\title{
Healthcare services utilization by respiratory tract infections patients attending primary healthcare centers in Qatar
}

Nazmul Islam

Qatar University

M Fasihul Alam ( $\Delta$ malam@qu.edu.qa )

Qatar University

Research article

Keywords: Respiratory tract infection, healthcare utilization, primary care

Posted Date: November 5th, 2019

DOI: https://doi.org/10.21203/rs.2.16854/v1

License: (c) (1) This work is licensed under a Creative Commons Attribution 4.0 International License.

Read Full License 


\section{Healthcare services utilization by respiratory tract infections patients attending primary healthcare centers in Qatar}

Nazmul Islam ${ }^{1}$, M. Fasihul Alam ${ }^{1 *}$

${ }^{1}$ Dept. of Public Health, College of Health Sciences, QU Health, Qatar University, Doha, Qatar

*Corresponding author:

M Fasihul Alam, $\mathrm{PhD}$

Department of Public Health

College of Health Science,

Qatar University, Doha, Qatar.

T: +974-44034784

Email addresses: malam@qu.edu.qa 


\section{Abstract}

Background: Respiratory-tract-infections (RTIs) are a common indication, and responsible for most of the healthcare services use at primary care. The study aimed to investigate the utilization and determinants of a number of healthcare services use by local Qatari patients presented at selected primary health centers in Qatar.

Methods: A retrospective study was conducted using an electronic database that captured RTIrelated healthcare services use from selected primary care health centers in Qatar during July 2015 to December 2017. Trends in monthly rates of healthcare services utilization were investigated via a linear regression, while patient-level count data on utilization were used to estimate a negative binomial regression to identify determinants of each of the four healthcare services use.

Results: In total, 171447 RTI-related visits were made by 58786 patients during July 2015 to December 2017. The mean age ( \pm SD) of participants is $24.80( \pm 18.5)$ years, and $48.91 \%$ of them are male. The highest number of patients with a comorbidity was diabetes (12.85\%), followed by hypertension (10.54\%). There was a small but statistically significant decreasing trend in the monthly rates of antibiotics consumption and an increasing trend in the rates of tests/investigations done, with less number of utilization during summer months. Healthcare services utilization varied significantly across age-categories and whether patients had one of the five comorbidities. Age and comorbidity status appeared to be important predictors for patient-level healthcare services utilization.

Conclusion: The study produces results on selected primary healthcare services utilization by local RTI patients, which are in line with evidence from the literature. It facilitates an evidence- 
base for policymakers to comprehend the scale of the problem and plan a better management of RTIs at primary care setting in Qatar.

\section{Keywords:}

Respiratory tract infection, healthcare utilization, primary care 


\section{Background:}

The World Health Organization (WHO) had declared antimicrobial resistance (AMR) as a global public health crisis that has reached at an alarming level (1). The widespread and inappropriate use of antibiotics in primary care has been considered as the most important risk factor for an infection with resistant organism (2). AMR appears to be responsible for prolonged severe infections, complications, extended hospital stays, more recurrent clinician visits and increased medicalization of self-limiting diseases (3). Along with an increased AMR, the excessive use of antibiotics may result in unexpected side effects and increase re-consultations for respiratory tract infections (RTIs) (4) (5). One of the most common reasons for General Practitioner (GP) visits and antibiotic use in primary care setting is acute RTIs, such as bronchitis, sinusitis, and rhinitis. RTIs are accounting for more than $60 \%$ of antibiotic use in primary care (6). Managing RTIs in younger children involves huge healthcare resources such as hospitalization, diagnostic tests and medicines (7). In the USA, almost $10 \%$ of ambulatory visits are related to the acute RTIs (8). However, antibiotics do not provide any clinical benefit to majority patients with RTIs $(4,9)$. Surveillance of antibiotic prescribing and its consumption is vital to manage increasing AMR globally.

It is evident that RTI related antibiotic use declined during 1990s, however, has remained unchanged ever since (10). In the UK, there has been a long-term decrease in the rate of general practitioner (GP) visits and antibiotic prescribing for managing respiratory infections (11). A study from Southern Sweden also indicated a reduction of $12 \%$ and $10 \%$ in yearly primary care consultation rates for acute tonsillitis and acute otitis media (AOM), respectively, between 1999 and 2005 (12). Over the years, Qatar has experienced a significant reduction in the proportion of deaths from infectious diseases but some infections like RTIs, AOM, intestinal infection and skin 
and soft tissue infection still remained high in the last decades, with a substantial impact on morbidity and mortality (13).

The UK national guidance on the self-limiting infection control suggests that most patients presenting with acute RTIs in primary care can be managed by either no or a delayed antibiotic prescribing, with prescriptions only being used if symptoms do not improve (14). A systematic review of treatment strategies for RTIs indicated that prescribing antibiotics for upper RTIs is not a suggested option, especially for acute laryngitis for which antibiotics such as penicillin $\mathrm{V}$ and erythromycin are not effective (15). Some non-Scandinavian countries suggested penicillin $\mathrm{V}$ as the first line treatment option and the European Society for Clinical Microbiology and Infectious Disease prescribed amoxicillin or an antibiotic medication to bringing down RTIs (16). However, no guideline has clearly indicated a particular antimicrobial as being the best in exact anti-infection treatment (17). Following national guidelines, a Canadian study investigated the GPs prescribing patterns for acute RTIs also recommended penicillin $\mathrm{V}$ as a first line antibiotic treatment for these infections (18).

In another systematic review, McKay et al. (19) identified a wide range of factors associated with antibiotic prescribing for RTIs. The authors found ten studies that explored Gender as a possible factor, and just one study (20) showed a statistically significant association between male sex and higher odds of antibiotic prescriptions. Nineteen studies explored age as a factor, ten studies suggested older population had higher odds/risk of prescriptions than younger and three studies suggested younger people had higher risks of antibiotic prescriptions. Nine studies in the review examined association between comorbidities and antibiotic prescribing for RTIs. Two of these studies found the presence of comorbidities was associated with antibiotic prescribing, however, the type of comorbidities was varied by studies. 
There is a limited evidence in the literature on healthcare services utilization for treating and managing RTIs in primary care setting in middle-eastern countries. A couple of studies on disease burden on health care services have been conducted in the region, however, no such studies done in Qatar. Therefore, the study aimed to investigate the trend, distribution and determinants of a number of healthcare services use (RTI-related visits, antibiotics and other medication use and tests and investigations) to manage RTIs among local Qatari patients presented at primary health care centers.

\section{Methods:}

\section{Study design and population:}

A retrospective observational study has been conducted using patient-level electronic data for RTI patients presented at the seven primary care health centers in Qatar. A complete dataset was available for selected centers from the CERNER electronic system over the study period, July 2015 to December 2017. Although there are in total 23 primary care health centers in Qatar, during the data analysis stage, the CERNER database had complete patient-level information for only seven primary healthcare centers over the study period. The data-availability issue from the electronic database in Qatar had also restricted the study duration to only being two and half years. Study population included Qatari patients of all ages attending selected primary care health centers for RTIs between July 2015 and December 2017. Diagnosis of RTIs has been done by either clinical assessment or by using diagnostic tests.

\section{Data:}

The CERNER electronic database provides patient-level information on age, gender, comorbidity status, RTI-related GP visits at primary healthcare centers, antibiotic and other medications use, 
tests and investigations done, etc. The recorded diagnosis in the database was considered as final, and not verified externally. Duplicate patients were excluded. Data were validated by the PHCC validation team by taking random sample from each month one encounter from each healthcare center. The RTI diagnoses for patients are based on the standard codes using the International Classification of Diseases, 10th Revision (ICD-10). RTI diagnosis includes both upper RTI i.e. acute tonsillitis, pharyngitis, laryngitis, otitis media and lower RTI i.e. acute bronchitis, pneumonia and whooping cough. The present study had both the PHCC (Ref No: PHCC/RS/17/10/013) and the Qatar University Institutional Review Board (IRB) (Ref No: QU-IRB 875-E/18) ethical approval for conducting the research.

\section{Statistical analysis:}

\section{Trends in monthly rates of four selected healthcare services use by RTI patients:}

Each of the four healthcare services use was converted into monthly rate and reported in per 1000 total all-cause visits. For example, monthly rate of RTI-related GP visit was calculated as:

$\frac{\text { Total number of RTI-related visits from all seven healthcare centers in a month }}{\text { Total number of all-cause visits from all seven healthcare ceneters in that month }} 1000$.

The same formula was applied to calculate monthly rates of RTI-related visit, antibiotic and other medication use and tests/investigations done for 30 months during July 2015 to December 2017. The trend in the monthly rate of each of the four selected healthcare services use over 30 months study period was presented in a graph. Since there is a likely seasonal influence on the occurrence of RTIs, hence an impact on the RTI-related healthcare services use, this was accounted for in the estimated regression model of the trend analysis. We estimated the following simple linear 
regression to investigate the trend in monthly rate of various RTI-related healthcare services used by these patients:

$\mathrm{Y}=a+b_{1}$ Month $+b_{2} \mathrm{Q} 2+b_{3} \mathrm{Q} 3+b_{4} \mathrm{Q} 4+e$

Where ' $\mathrm{Y}$ ' is the dependent variable (monthly utilization rate for one of the 4 healthcare services use), 'Month' represents time-trend of 30 months of the study period (1-30, where ' 1 ' for July 2015, '2' for August 2015, ......, '30' for December 2017). Quarters 'Q1' to 'Q4' are seasonal dummies, where 'Q1' refers to January to March (Jan. to Mar.=1, else=0), 'Q2' refers to April to June (Apr. to Jun. =1, else=0), 'Q3' refers to July to September (Jul. to Sept. =1, else=0) and 'Q4' refers to October to December (Oct. to Dec. $=1$, else=0) of a year, 'Q1' is a reference category and $e$ represents the random error. In the fitted regression model, which is adapted from Alam et al. (21), $b_{1}$ estimates the monthly trend in the dependent variable (' $\mathrm{Y}$ ') after adjusting for seasonal effects, and $b_{2}, b_{3}$ and $b_{4}$ are the regression coefficients for the respective seasonal dummies.

\section{Analysis of four selected healthcare services use at individual patient-level:}

We also analysed individual patient-level data on RTI-related four selected healthcare services use. Patient-level total number of RTI-related visits, antibiotic use, other medication use, and tests and investigations were calculated for seven healthcare centers over the study period. We investigated whether a number of patient-level demographic and clinical characteristics (that were available from the CERNER database, e.g. age categories, gender and whether the patient had any of the medical conditions such as diabetes, hypertension, asthma, dyslipidaemia or obesity) determine the utilization of the healthcare services. Because of the non-normality of the outcome variables, we applied non-parametric Kruskal-Wallis and Mann-Whitney U tests (as appropriate) 
and reported the p-values to show the significance of results from a bi-variate analysis between a dependent (each of the four healthcare services) and any of the independent variables.

The study also estimated a negative binomial multiple regression model to identify determinants of each of the four healthcare services use, where the choice of a negative binomial model is due to over-dispersed count outcome variables. All independent variables which showed a p-value $<0.25$ in bi-variate analyses, along with the variables of known clinical importance, were included in the initial full regression model. The significance of a variable was assessed using its p-value, and variables that did not make contribution to the model (with $p$-value $>0.05$ ) were eliminated and a new smaller model fitted. These two models were then compared by using Likelihood Ratio (LR) chi-square test to ensure that a better model is estimated with a smaller log-likelihood value.

Regression coefficients of the negative binomial model parameters were interpreted as the difference between log of expected counts or incidence rate ratios (IRR).

All statistical analyses were two-sided, and a $p$-value less than 0.05 considered to be significant. Analyses were done by using the STATA statistical software package 15.0.

\section{Results:}

A total of 171447 RTI-related visits made by 58786 patients during July-2015 to December-2017. The Mean age \pm SD of patients is $24.80 \pm 18.5$ years, among them $38.79 \%$ falls to age group $5-19$ and $33.53 \%$ belongs to $20-44$. Of the total patients, $48.91 \%$ are male and the rest are female. Highest number of patients with a comorbidity of Diabetes (12.85\%), followed by Hypertension $(10.54 \%)$ (Table 1). 
( Table 1 here)

Table-2 presents results from the estimated regression model (i) for the trend analyses in monthly rates of four selected healthcare services use. Monthly rates of RTI-related GP visits during July 2015 and December 2017 decreased by 0.5 visit (per 1000 total all-cause visits) which was not statistically significant $(\mathrm{p}=0.441)$. However, the rate of RTI-related antibiotic use was decreased significantly by about 0.8 visit (per 1000 total all-cause visits) over the study period ( $<<0.001)$. Although for both other medications use and tests and investigations there was a slightly increasing trend in the monthly rates, it was statistically significant for tests/investigations $(\mathrm{p}<0.001)$ whereas for other medications use the trend was not significant $(\mathrm{p}=0.797)$. As expected, RTI-related GP visits were significantly lower in Quarters 2 and 3 (Q2, Q3) compare to Q1 of winter months (Jan.Mar.)

(Table 2 here)

Figures 1-4 depict the trend in the monthly rate of four selected healthcare services use by RTI patients from July 2015 to December 2017. It is clear from these figures that, although there was no difference in the month-to-month rates of RTI-related GP visits over the study period, the figures revealed a decreasing trend in the monthly rate of RTI-related antibiotics consumption and an increasing trend in the tests/investigations done (Figures 2 and 4, respectively). Figure 3 does not show a clear trend in the monthly rates of other medications use.

(Figures 1 to 4 here)

A bi-variate analysis investigated association between individual patient-level number of RTIrelated GP visits, antibiotic use, other medications use and tests/investigations across agecategories, gender and various comorbidity status. The results indicated that RTI-related GP visits, 
antibiotic use, other medications use and tests/investigations varied significantly (p-value $<0.001$ ) across age-categories and whether or not patients had one of the five comorbidities (Appendix 1).

Results from the negative binomial regression model for each of the four selected healthcare services use revealed that, age, gender and five comorbidity status were significantly associated with patient-level RTI-related GP visits. Patients aged 20 and above had significantly lower RTIrelated GP visits than younger population during the study period. Male RTI patients had 2\% more GP visits $(\mathrm{p}=0.01)$ than female counterparts. RTI patients with asthma had $46 \%$ higher GP visits than those had no asthma ( $<<0.001)$, similarly RTI patients with diabetes had 8\% higher GP visits than those without diabetes $(\mathrm{p}<0.001)$. Hypertension, dyslipidaemia and obesity also appeared to be significantly affecting RTI-related GP visits (Table 3).

(Table 3 here)

Age, diabetes, asthma and obesity were found to be significantly associated with patient-level RTIrelated antibiotic use over the study period. Patients aged between 5 to 19 years and 20 to 49 years experienced $10 \%$ and $26 \%$ reduction, respectively, in antibiotic use than those aged less than 5 years $(\mathrm{p}<0.001)$. RTI patients with diabetes had 9\% increase in antibiotic use than those without diabetes $(\mathrm{p}<0.001)$, similarly patients with asthma had $46 \%(\mathrm{p}<0.001)$ and obesity had $16 \%$ $(\mathrm{p}<0.001)$ increase in antibiotic use than those without asthma and obesity, respectively. Age and asthma also appeared to be significant determinants of healthcare services utilization in terms of 'other medication use' and 'tests \& investigations'. For example, RTI patients with asthma experienced $42 \%$ increase in RTI-related tests and investigations than those without asthma $(\mathrm{p}<0.001)($ Table 3). 


\section{Discussion:}

The study observed higher rates of RTI-related GP visits, antibiotics and other medications use and tests/investigations (per 1000 all-cause visits) in young Qatari patient's age between 5 to 19 years. Among patients with at least one of the five comorbidities, the highest rates of all four healthcare services utilization by RTI patients with diabetes. Our study showed that, overall, there was a non-significant decreasing trend in RTI-related monthly rates of GP visits during July 2015 to December 2017. However, the monthly rates of antibiotics used by RTI patients decreased significantly but in small amount (one antibiotic per 1000 all-cause visits) during the study period. For RTI-related tests and investigations, there was a significant increasing trend in rates, but in small amount. Higher usage of tests and investigations may be due to suppliers' induced demand for unnecessary health care services generated in a private health care insurance market like in Qatar. In addition, the local Qatari RTI patients have zero out-of-pocket costs towards their tests and investigations, which, in many cases might not be driven by needs based on clinical assessments.

These findings have been in line with results from other studies in the literature, showing a decreasing trend in GP visits and antibiotic use by RTI patients (22), (23). In the UK, there has been a long-term decreasing trend in the rate of GP consultations and antibiotic prescriptions for managing respiratory infections (11). The reduction in RTI-related GP consultations was also observed in a Swedish study, which revealed a $10-12 \%$ reduction in yearly primary care consultation rates for acute tonsillitis between 1999 and 2005 (24). Our findings reinforce the importance of a national level antimicrobial stewardship program in relation to appropriate use of antibiotics by reducing unnecessary RTI-related GP visits and antibiotic consumptions (25),(26). 
The study indicated that age and comorbidity status (whether RTI patients had a comorbidity or not) are important predictors for determining individual patient-level health care services use. For the RTI related GP visits and other medications use the predictors were appeared to be similar i.e. age, diabetes mellitus, hypertension, asthma, dyslipidemia, and obesity. In case of RTI related antibiotic use - age, diabetes mellitus, asthma and obesity were identified as significant predictors, and for RTI related tests \& investigations only age and asthma were appeared to be as significant determinants. These findings were in line with other studies in the literature, for example, in a systematic review McKay et al. (19) investigated a wide range of factors associated with antibiotic prescribing for RTIs. Nineteen studies examined age as a factor, 10 suggested older people had higher odds/risks of prescriptions than younger population and 3 suggested younger people had higher risks of antibiotic prescriptions. Nine studies in the review investigated associations between comorbidities and antibiotic prescribing for RTIs. Two of these studies found the presence of comorbidities was associated with prescribing, however, the type of comorbidities was varied by studies (19),(20).

As expected, the study findings revealed a lower number of RTI related resource use during the summer months. In general, the new event of RTIs is higher in the winter months. The population dynamics in the Gulf Cooperation Council (GCC) states are such that a large number of migrant workforces return to their home states during the summer break, and the Qatari nationals travel overseas for leisure. A smaller Qatari population would lead to a smaller denominator of persons looking for treatment, and a smaller overall population may be related with lower transmission rates. Whether the lower figures in Qatar during the summer months are due to the usual epidemiological patterns seen everywhere in the world, or to a much lesser populace base in those months, warrant further investigation (27). However, the results showed that there was a 
substantial seasonal variation in RTI-related GP visits and antibiotic use, an indication of higher utilization of these services (12).

This is a first ever study in Qatar which has investigated the trend and distribution of primary healthcare service utilization across different subgroups of RTI patients. Based on appropriate statistical methods, study findings are indicative, clinically meaningful, and in line with those observed in the literature. The study facilitates a broader assessment of RTI-related healthcare services utilization both from a provider and a patient perspective. It has used the national level electronic database which provides complete and valid routine observational data from primary care centers in Qatar.

We acknowledge a number of limitations of the study. There is a total of twenty-three primary care health centers in Qatar, but during the data collection the CERNER electronic database was incomplete, and had complete patient-level information for only seven healthcare centers over the study period. The study assumed that it will not substantially affect its findings since the selected healthcare centers are the largest in terms of number of the registered patients and cover all geographical regions in Qatar. Selection of study-period was also restricted to two and half-years due to data availability issue. Another limitation of the study is that, it could not include non-Qatari population since local policy makers and the sponsor who provided study-data was interested in research focusing local population for whom the PHCC electronic database was up to date. The study couldn't capture RTI-related healthcare services at hospitals due to data linkage and availability issues, hence focused only on selected health care utilizations at primary care level. The current study used "all cause visits" (as denominator) in the calculation of relative measure of monthly utilization rate. It could be argued that, without knowing the nature of non-RTI related 'other causes'- the impact of which could be fixed or changing over the time, the observed trend 
in healthcare service utilization might be misleading. Nonetheless, this issue could be handled by considering the total number of registered residents as the denominator of the equation. However, the current practice allows local Qatari people to receive their required healthcare services at any primary health centers in Qatar, means that, there is no 'fixed' number of registered local residents for a primary health center. Hence, to avoid likely duplication in data, the study could not use 'total number of registered residents' as a potential denominator in the calculation of the outcome measure via monthly utilization rate.

The tests/investigations data used in the study also raised an issue, whether these services were used only to diagnose RTI or other underlying conditions. Once a patient is diagnosed with an RTI, all tests and investigations done in that visit are recorded as 'RTI-related' services. However, in reality there could be some tests and investigations, which might not exactly be related to an RTI. It was not possible to separate those non-RTI related tests and investigations data from the analyses, as the database provided tests and investigations data as a count variable without a detail information on data. This study only used five comorbidity conditions but couldn't capture other comorbidities i.e. cancer, COPD and HIV, etc. due to the limitation of the data-availability. Also, to note that, as an observational study it had no intention to intervene the on-going treatment, thus the final diagnoses registered for each patient were not confirmed by results from microbiology or from culture and sensitivity tests. The absence of results from microbiology and culture and sensitivity tests will make it difficult to decide whether diagnosis or treatment was rational or not, and misclassification of diagnosis may lead to overestimation or underestimation of the utilization. 


\section{Conclusions}

The evidence produced from the study facilitates to guide an effective and efficient management of healthcare services needed to manage RTI patients presented at primary care settings in Qatar. Although there has been a decreasing trend in the usage of a number of healthcare services, it is likely that unnecessary tests and investigations are utilized by RTI patients that need further attention. The findings of the study should be interpreted with caution; however, the authors argue that the study produces an evidence-base for policymakers to comprehend the scale of the burden and to plan accordingly for the better management of RTIs.

\section{Authors' contributions}

MFA conceived and led the study design as a Master of Public Health (MPH) thesis for NI. NI contributed to data collection, and conducted the main data analysis under the supervision of MFA. Statistical analysis plan was designed by MFA. Both authors contributed to prepare the main draft, provided critical review and approved the final manuscript. 


\section{Abbreviations}

AMR-Antimicrobial Resistance; AOM - Acute Otitis Media; GCC- Gulf Cooperation Council; GP-General Practitioner; ICD - International Classification of Diseases; IRB- Institutional Review Board; IRR- Incidence Rate Ratio; LRT- Likelihood Ratio Test; PHCC- Primary Health Care Corporation; QU- Qatar University; RTI- Respiratory Tract Infection; SD- Standard Deviation; UK- United Kingdom; USA - United States of America; WHO- World Health Organization

\section{Ethics Approval and Consent to Participate}

The study had ethics approval from the Primary Health Care Corporation (PHCC), Al Mina Street, Doha, PO Box no. 26555, Qatar (Ref No: PHCC/RS/17/10/013) and from the Qatar University Institutional Review Board (IRB) (Ref No: QU-IRB 875-E/18) for conducting the research. Since the study used a secondary database provided by the PHCC, a 'consent to participate' is not applicable.

\section{Competing interests}

The authors declare no conflict of interests.

\section{Declarations}

Part of the study was funded by the Qatar University internal student grant no. QUST-1-CHS2018-5 in terms of purchasing a software (STATA) for data analysis. Supporting data can be accessed via the corresponding author upon reasonable request. 


\section{Acknowledgements}

The authors greatly acknowledge the support from the Primary Health Care Corporation (PHCC) in Qatar for providing necessary data for the study. We are also grateful to Department of Public Health, Qatar University faculties for their comments and suggestions while the second author presented some of the study findings during his MPH thesis internal presentation. 


\section{References}

1. World Health O. The evolving threat of antimicrobial resistance: options for action: Geneva: World Health Organization; 2012.

2. Costelloe C, Metcalfe C, Lovering A, Mant D, Hay AD. Effect of antibiotic prescribing in primary care on antimicrobial resistance in individual patients: systematic review and metaanalysis. BMJ. 2010;340:c2096.

3. Llor C, Bjerrum L. Antimicrobial resistance: risk associated with antibiotic overuse and initiatives to reduce the problem. Ther Adv Drug Saf. 2014;5(6):229-41.

4. Little P, Gould C, Williamson I, Warner G, Gantley M, Kinmonth AL. Reattendance and complications in a randomised trial of prescribing strategies for sore throat: the medicalising effect of prescribing antibiotics. BMJ. 1997;315(7104):350-2.

5. Venekamp RP, Sanders SL, Glasziou PP, Del Mar CB, Rovers MM. Antibiotics for acute otitis media in children. Cochrane Database Syst Rev. 2015;2015(6).

6. Petersen I, Hayward AC. Antibacterial prescribing in primary care. J Antimicrob Chemother. 2007;60(suppl_1):i43-i7.

7. Lara-Oliveros CA, De Graeve D, Franco F, Daza SP. Disease burden and medical costanalysis of Acute Respiratory Infections in a low-income district of Bogotá. Revista de Salud Pública. 2016;18(4):568-80.

8. Grijalva CG, Nuorti JP, Griffin MR. Antibiotic prescription rates for acute respiratory tract infections in US ambulatory settings. JAMA. 2009;302(7):758-66.

9. Macfarlane J, van Weel C, Holmes W, Gard P, Thornhill D, Macfarlane R, et al. Reducing antibiotic use for acute bronchitis in primary care: blinded, randomised controlled trial of patient information leafletCommentary: More self reliance in patients and fewer antibiotics: still room for improvement. BMJ. 2002;324(7329):91-4.

10. Ashworth M, Cox K, Latinovic R, Charlton J, Gulliford M, Rowlands G. Why has antibiotic prescribing for respiratory illness declined in primary care? A longitudinal study using the General Practice Research Database. J Public Health. 2004;26(3):268-74. 
11. Gulliford M, Latinovic R, Charlton J, Little P, van Staa T, Ashworth M. Selective decrease in consultations and antibiotic prescribing for acute respiratory tract infections in UK primary care up to 2006. J Public Health. 2009;31(4):512-20.

12. Neumark T, Brudin L, Mölstad S. Antibiotic prescribing in primary care by international medical graduates and graduates from Swedish medical schools. J Fam Pract. 2015:cmv001.

13. Khamis FA, Al-Kobaisi MF, Al-Areimi WS, Al-Kindi H, Al-Zakwani I. Epidemiology of respiratory virus infections among infants and young children admitted to hospital in Oman. J Med Virol. 2012;84(8):1323-9.

14. Tan T, Little P, Stokes T. Antibiotic prescribing for self limiting respiratory tract infections in primary care: summary of NICE guidance. BMJ. 2008;337:a437.

15. Reveiz L, Cardona AF. Antibiotics for acute laryngitis in adults. The Cochrane Library. 2015.

16. Woodhead M, Blasi F, Ewig S, Garau J, Huchon G, Ieven M, et al. Guidelines for the management of adult lower respiratory tract infections-Full version. Clin Microbiol Infect. 2011;17(s6).

17. Pakhale S, Mulpuru S, Verheij TJM, Kochen MM, Rohde GGU, Bjerre LM. Antibiotics for community-acquired pneumonia in adult outpatients. The Cochrane Library. 2014.

18. Gjelstad S, Straand J, Dalen I, Fetveit A, Strøm H, Lindbæk M. Do general practitioners' consultation rates influence their prescribing patterns of antibiotics for acute respiratory tract infections? J Antimicrob Chemother. 2011;66(10):2425-33.

19. McKay R, Mah A, Law M, McGrail K, Patrick DM. Systematic review of factors associated with antibiotic prescribing for respiratory tract infections. Antimicrob Agents Chemother. 2016:AAC-00209.

20. Kozyrskyj AL, Dahl ME, Chateau DG, Mazowita GB, Klassen TP, Law BJ. Evidencebased prescribing of antibiotics for children: role of socioeconomic status and physician characteristics. Can Med Assoc J. 2004;171(2):139-45. 
21. Alam MF, Cohen D, Dunstan F, et al. Impact of the phased abolition of co-payments on the utilisation of selected prescription medicines in Wales, Health Econ. 2018, 27(1): 236-243.

22. Garcell HG, Arias AV, Fernandez EA, Guerrero YB, Serrano RNA. Antibiotic consumption during a 4-year period in a community hospital with an antimicrobial stewardship program. Oman Med J. 2016;31(5):352.

23. Ashworth M, Charlton J, Ballard K, Latinovic R, Gulliford M. Variations in antibiotic prescribing and consultation rates for acute respiratory infection in UK general practices 19952000. Br J Gen Pract. 2005;55(517):603-8.

24. Tyrstrup M, Beckman A, Mölstad S, Engström S, Lannering C, Melander E, et al. Reduction in antibiotic prescribing for respiratory tract infections in Swedish primary care-a retrospective study of electronic patient records. BMC Infect Dis. 2016;16(1):709.

25. Butler CC, Simpson SA, Dunstan F, Rollnick S, Cohen D, Gillespie D, et al. Effectiveness of multifaceted educational programme to reduce antibiotic dispensing in primary care: practice based randomised controlled trial. BMJ. 2012;344:d8173.

26. Wu C-T, Chen C-L, Lee H-Y, Chang C-J, Liu P-Y, Li C-Y, et al. Decreased antimicrobial resistance and defined daily doses after implementation of a clinical culture-guided antimicrobial stewardship program in a local hospital. J Microbiol Immunol Infect. 2017;50(6):846-56.

27. Butt AA, Navasero CS, Thomas B, Al Marri S, Al Katheeri H, Al Thani A, et al. Antibiotic prescription patterns for upper respiratory tract infections in the outpatient Qatari population in the private sector. Int J Infect Dis. 2017;55:20-3. 
Table-1: Descriptive of all RTI patients $(\mathrm{N}=58,786)$ attended primary healthcare centers in Qatar during July 2015 to December 2017.

\begin{tabular}{|c|c|c|}
\hline Variables & & $\begin{array}{l}\text { Continuous data; } \text { Mean } \pm \text { SD } \\
\text { Categorical data; } \text { Number }(\%)\end{array}$ \\
\hline Age & $($ Mean \pm SD) & $(24.80 \pm 18.5)$ \\
\hline \multirow{4}{*}{ Age-category } & $<5$ years $\mathrm{N}(\%)$ & $6,163(10.48)$ \\
\hline & $5-19$ years $\mathrm{N}(\%)$ & $22,803(38.79)$ \\
\hline & $20-44$ years $\mathrm{N}(\%)$ & 19,709 (33.53) \\
\hline & $45+$ years $\mathrm{N}(\%)$ & $10,111(17.20)$ \\
\hline \multicolumn{3}{|l|}{ Gender } \\
\hline & Male N (\%) & $28,753(48.91)$ \\
\hline & Female N (\%) & $30,033(51.09)$ \\
\hline \multicolumn{3}{|l|}{ Diabetes } \\
\hline & Yes N (\%) & $7,555(12.85)$ \\
\hline & No N (\%) & $51,231(87.15)$ \\
\hline \multicolumn{3}{|l|}{ Hypertension } \\
\hline & Yes N (\%) & $6,195(10.54)$ \\
\hline & No N $(\%)$ & $52,591(89.46)$ \\
\hline \multicolumn{3}{|l|}{ Asthma } \\
\hline & Yes N (\%) & $4,293(7.30)$ \\
\hline & No N (\%) & $54,493(92.70)$ \\
\hline \multicolumn{3}{|l|}{ Dyslipidemia } \\
\hline & Yes N (\%) & $3,481(5.92)$ \\
\hline & No N (\%) & $55,305(94.08)$ \\
\hline \multicolumn{3}{|l|}{ Obesity } \\
\hline & Yes N (\%) & $2,743(4.67)$ \\
\hline & No N $(\%)$ & $56,043(95.33)$ \\
\hline
\end{tabular}


Table-2: Trend analysis using estimated regression model (1) for monthly rates of four selected healthcare services use by RTI patients during July 2015 to December 2017.

\begin{tabular}{|c|c|c|c|c|c|}
\hline RTI-related GP visits & Estimates & $P$-value & \multicolumn{2}{|c|}{ 95\% Confidence Interval } & Adjusted-R ${ }^{2}$ \\
\hline Months & -0.47 & 0.441 & -1.70 & 0.76 & 0.69 \\
\hline Q1 (Jan. to Mar.) & Reference & & & & \\
\hline Q2 (Apr. to Jun.) & -47.87 & 0.007 & -81.30 & -14.45 & \\
\hline Q3 (Jul. to Sept.) & -63.14 & $<0.001$ & -93.47 & -32.81 & \\
\hline Q4 (Oct. to Dec.) & 26.76 & 0.083 & -3.79 & 57.31 & \\
\hline $\begin{array}{l}\text { RTI-related } \\
\text { Antibiotic use }\end{array}$ & Estimates & $P$-value & \multicolumn{2}{|c|}{ 95\% Confidence Interval } & Adjusted- $R^{2}$ \\
\hline Months & -0.84 & $<0.001$ & -1.26 & -0.43 & 0.64 \\
\hline Q1 (Jan. to Mar.) & Reference & & & & \\
\hline Q2 (Apr. to Jun.) & -5.77 & 0.298 & -16.97 & 5.42 & \\
\hline Q3 (Jul. to Sept.) & -15.64 & 0.004 & -25.79 & -5.48 & \\
\hline Q4 (Oct. to Dec.) & 8.66 & 0.093 & -1.57 & 18.89 & \\
\hline $\begin{array}{l}\text { Other Medications } \\
\text { use }\end{array}$ & Estimates & $P$-value & \multicolumn{2}{|c|}{ 95\% Confidence Interval } & Adjusted-R ${ }^{2}$ \\
\hline Months & 0.50 & 0.797 & -3.50 & 4.51 & 0.57 \\
\hline Q1 (Jan. to Mar.) & Reference & & & & \\
\hline Q2 (Apr. to Jun.) & -103.62 & 0.060 & -212.15 & 4.91 & \\
\hline Q3 (Jul. to Sept.) & -127.71 & 0.013 & -226.17 & -29.24 & \\
\hline Q4 (Oct. to Dec.) & 95.72 & 0.058 & -3.48 & 194.91 & \\
\hline Tests/Investigations & Estimates & $P$-value & \multicolumn{2}{|c|}{ 95\% Confidence Interval } & Adjusted-R ${ }^{2}$ \\
\hline Months & 0.49 & $<0.001$ & 0.33 & 0.64 & 0.65 \\
\hline Q1 (Jan. to Mar.) & Reference & & & & \\
\hline Q2 (Apr. to Jun.) & -1.69 & 0.410 & -5.83 & 2.46 & \\
\hline Q3 (Jul. to Sept.) & -0.37 & 0.843 & -4.12 & 3.39 & \\
\hline Q4 (Oct. to Dec.) & 0.71 & 0.703 & -3.08 & 4.50 & \\
\hline
\end{tabular}


Table 3: Estimated negative binomial regression for patient-level four healthcare services utilization by RTI patients during July 2015 to December 2017

\begin{tabular}{|c|c|c|c|c|c|c|c|c|}
\hline \multirow[t]{2}{*}{ Variables } & \multicolumn{2}{|c|}{ GP Visits } & \multicolumn{2}{|c|}{ Antibiotic use } & \multicolumn{2}{|c|}{$\begin{array}{c}\text { Other } \\
\text { Medication }\end{array}$} & \multicolumn{2}{|c|}{$\begin{array}{c}\text { Tests \& } \\
\text { Investigations }\end{array}$} \\
\hline & IRR & $\begin{array}{l}p- \\
\text { value }\end{array}$ & IRR & $p$-value & IRR & $\begin{array}{l}p- \\
\text { value }\end{array}$ & IRR & $p$-value \\
\hline \multicolumn{9}{|l|}{ Age category } \\
\hline$<5$ years & $\begin{array}{l}1 \\
\text { (Ref.) }\end{array}$ & & 1(Ref.) & & 1(Ref.) & & 1(Ref.) & \\
\hline 5 to 19 years & 0.98 & 0.31 & 0.90 & $<0.001$ & 0.91 & $<0.001$ & 0.76 & $<0.001$ \\
\hline 20 to 44 years & 0.76 & $<0.001$ & 0.64 & $<0.001$ & 0.68 & $<0.001$ & 0.52 & $<0.001$ \\
\hline $45+$ years & 0.71 & $<0.001$ & 0.59 & $<0.001$ & 0.62 & $<0.001$ & 0.39 & $<0.001$ \\
\hline \multicolumn{9}{|l|}{ Gender } \\
\hline Female & $\begin{array}{l}1 \\
\text { (Ref.) }\end{array}$ & & & & & & & \\
\hline Male & 1.02 & 0.01 & & & & & & \\
\hline \multicolumn{9}{|l|}{$\begin{array}{l}\text { Diabetes } \\
\text { Mellitus }\end{array}$} \\
\hline No & $\begin{array}{l}1 \\
\text { (Ref.) }\end{array}$ & & 1 (Ref.) & & 1 (Ref.) & & & \\
\hline yes & 1.08 & $<0.001$ & 1.09 & 0.017 & 1.08 & $<0.001$ & & \\
\hline \multicolumn{9}{|l|}{ Hypertension } \\
\hline No & $\begin{array}{l}1 \\
\text { (Ref.) }\end{array}$ & & & & 1 (Ref.) & & & \\
\hline yes & 1.14 & $<0.001$ & & & 1.14 & $<0.001$ & & \\
\hline \multicolumn{9}{|l|}{ Asthma } \\
\hline No & $\begin{array}{l}1 \\
\text { (Ref.) }\end{array}$ & & 1 (Ref.) & & 1 (Ref.) & & 1 (Ref.) & \\
\hline yes & 1.46 & $<0.001$ & 1.46 & $<0.001$ & 1.47 & $<0.001$ & 1.42 & $<0.001$ \\
\hline \multicolumn{9}{|l|}{ Dyslipidemia } \\
\hline No & $\begin{array}{l}1 \\
\text { (Ref.) }\end{array}$ & & & & 1 (Ref.) & & & \\
\hline yes & 1.11 & $<0.001$ & & & 1.10 & $<0.001$ & & \\
\hline \multicolumn{9}{|l|}{ Obesity } \\
\hline No & $\begin{array}{l}1 \\
\text { (Ref.) }\end{array}$ & & 1 (Ref.) & & 1 (Ref.) & & & \\
\hline yes & 1.09 & $<0.001$ & 1.16 & $<0.001$ & 1.11 & $<0.001$ & & \\
\hline
\end{tabular}




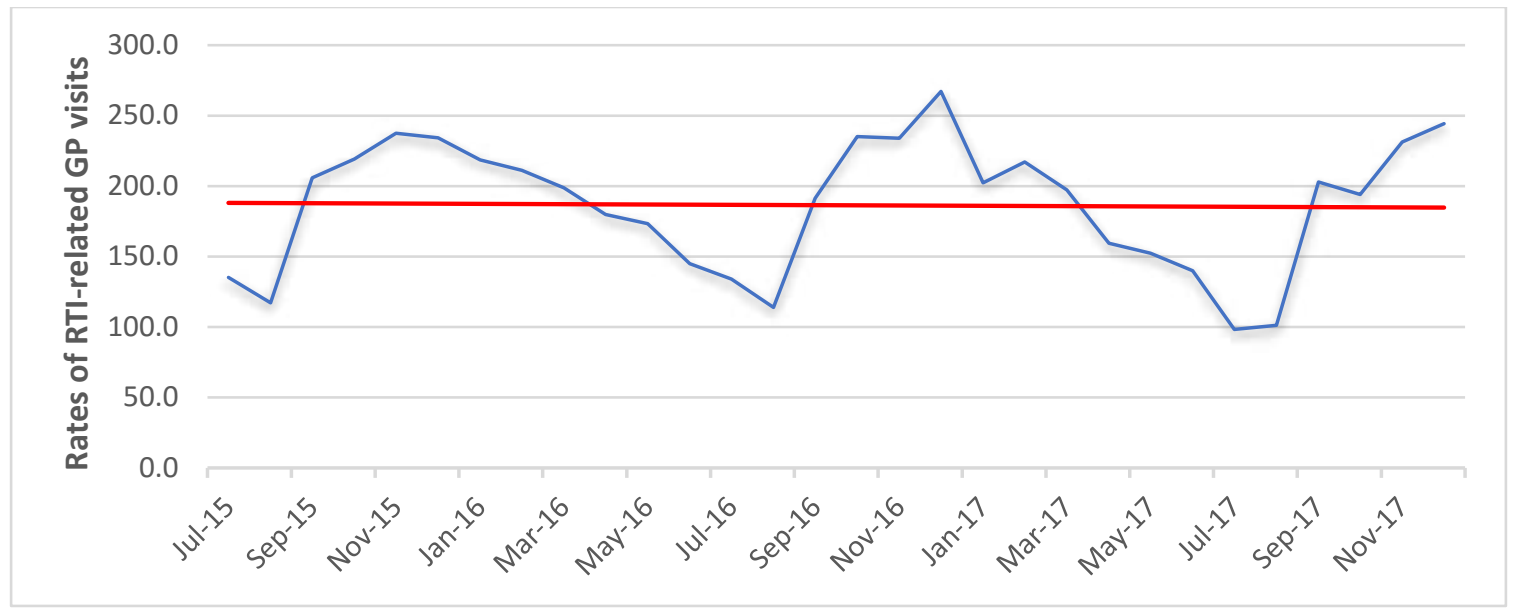

Figure 1: Monthly rate of RTI-related GP visits (per 1000 total all-cause visits) during July 2015 to December 2017. 


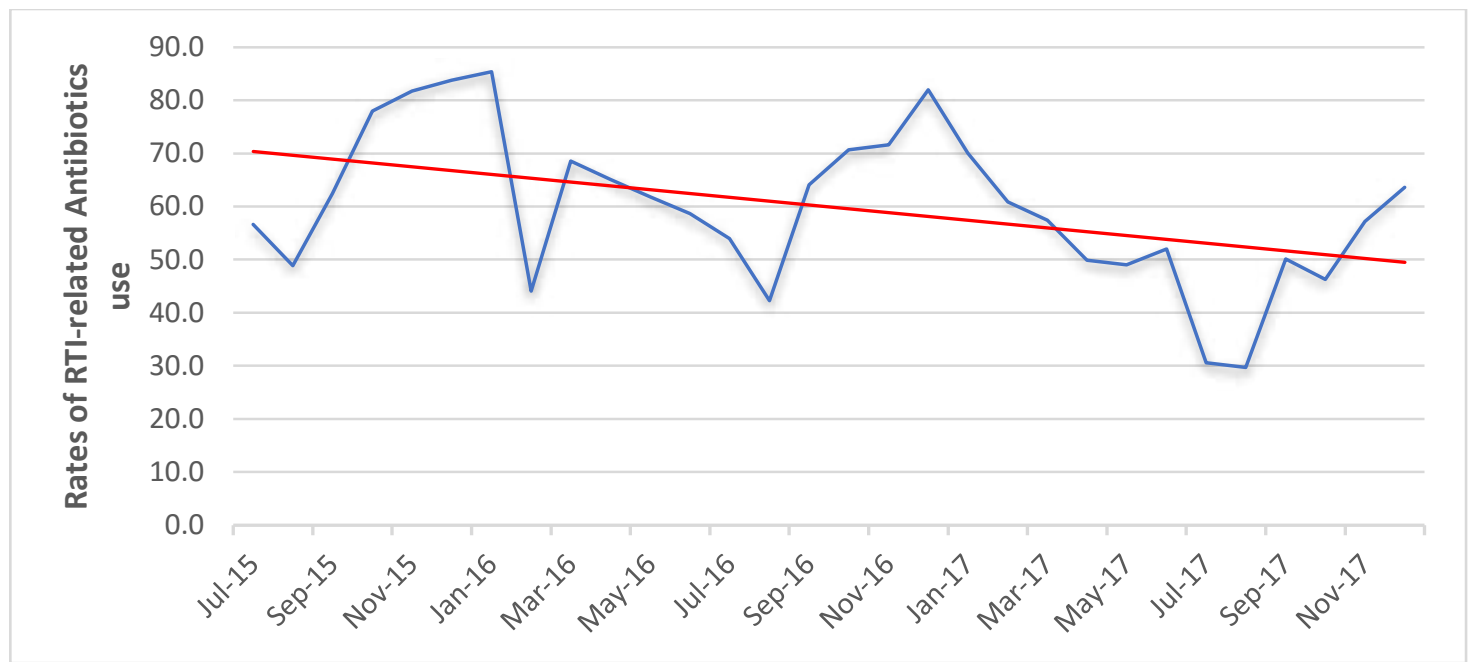

Figure 2: Monthly rate of RTI-related antibiotics use (per 1000 total all-cause visits) during July 2015 to December 2017. 


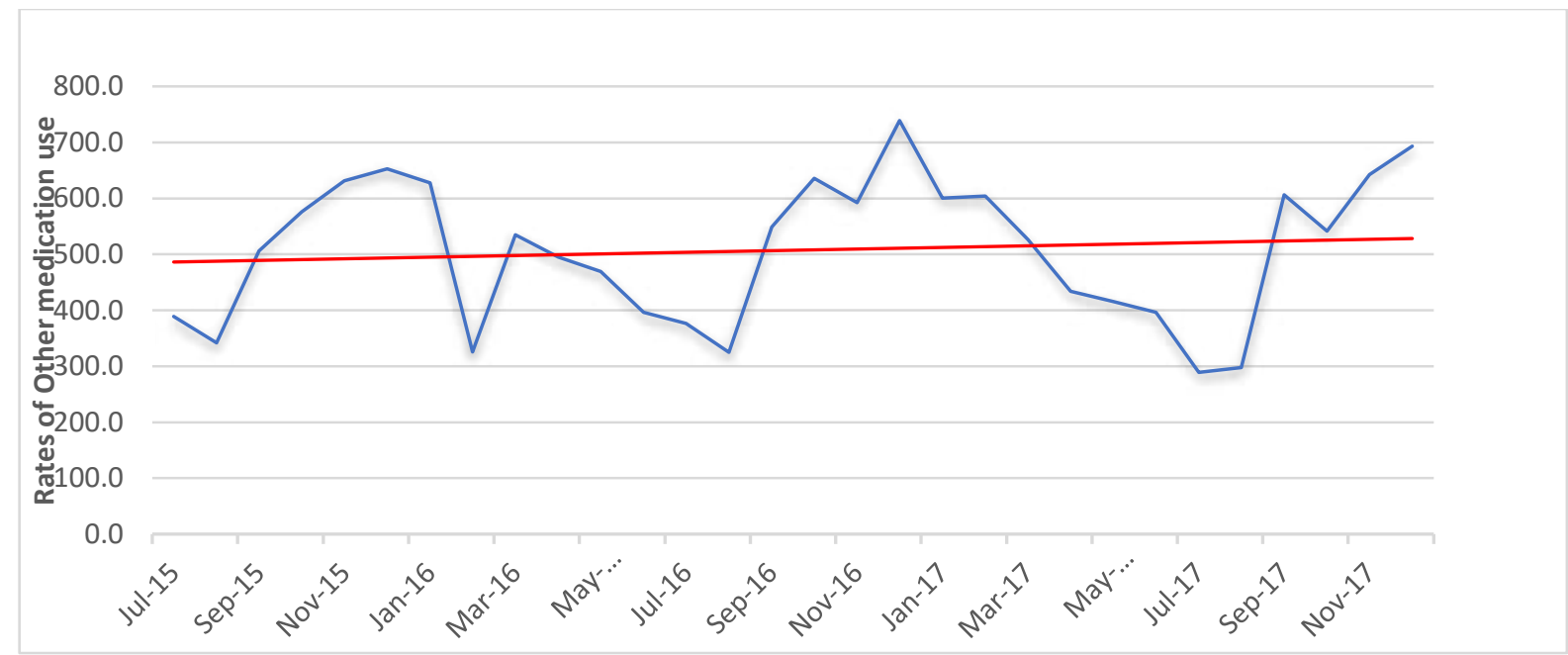

Figure 3: Monthly rate of RTI-related other medication use (per 1000 total all-cause visits) during July 2015 to December 2017. 


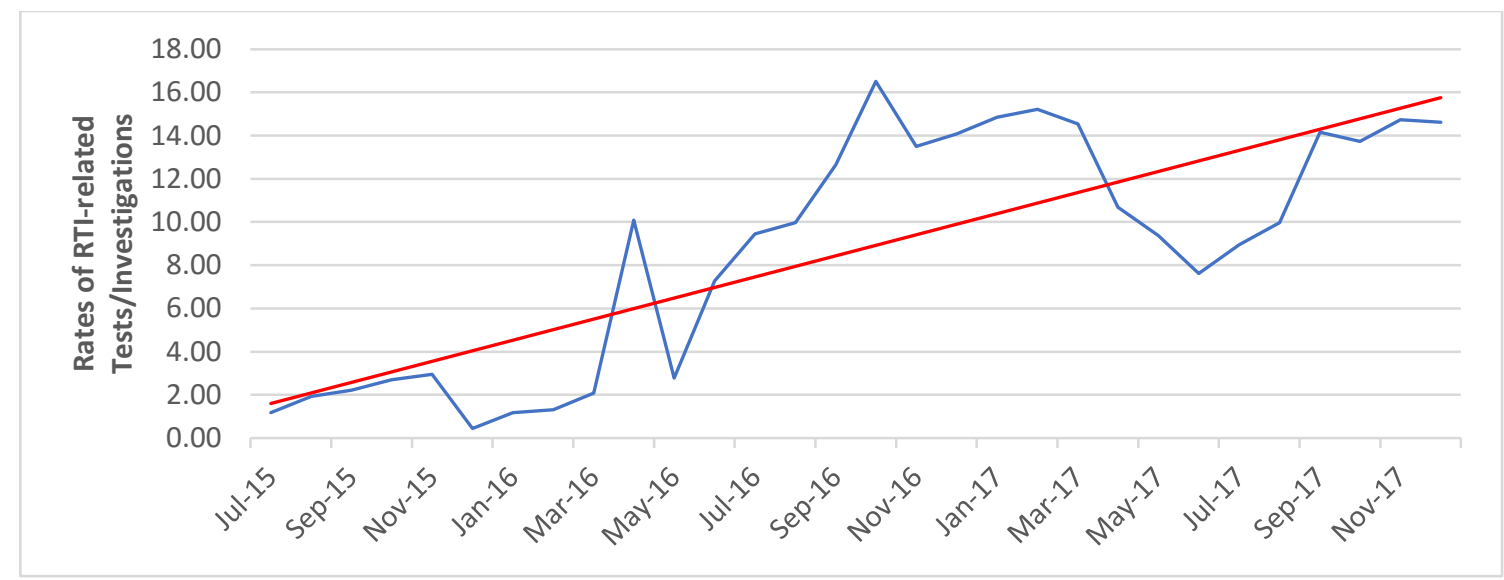

Figure 4: Monthly rate of RTI-related tests/investigations use (per 1000 total all-cause visits) during July 2015 to December 2017 